\title{
Bridging the Digital Divide by Building and Developing Wireless Community Networks in Rural Areas of Sri Lanka
}

\author{
Chandima H. de Silva \\ Department of Statistics \& Computer Science \\ University of Keleniya, Kelaniya \\ Revantha Udugampola \\ David Pieris Information Technologies Ltd \\ Colombo
}

\begin{abstract}
This paper discusses how new and emerging wireless technologies could be deployed together with Open Source product lines in expediting the propagation of the ICT know-how in the rural as well as the suburban areas of Sri Lanka. The intention of the authors is to showcase a blueprint for using newer and emerging viable communication technologies in the rural context. Current internet distribution networks generally rely on a permanent, fixed and largely wired infrastructure that is owned and often operated by large entities such as telecom operators or an Internet Service Provider. A relatively new and emerging technology is wireless Ethernet or wireless networking using the IEEE 802.11 standard. This standard encompasses the lower layers of the OSI model for transport of data as Ethernet frames using a spread spectrum based radio link. This technology opens the possibility of building a network without having the problems associated with, and the cost of putting some sort of physical transmission medium in the ground. Instead, antennas can be used to send and receive data using radio waves through free air. Due to the relative simplicity of the currently available commodity hardware that uses IEEE 802.11 technology it is relatively easy to build a local wireless community network in a community township. Using this network people can share resources among each other and, more importantly, it would facilitate the adaptation of a highly collaborative environment forgoing the language barriers, which is regarded as a major cause of the ethnic conflict in Sri Lanka.
\end{abstract}

\section{Introduction}

Current computer networks generally rely on a permanent, fxed and largely wired infrastructure which is owned and often operated by large entities such as telecom operators. A relatively new and emerging technology is wireless ethernet or wireless networking using the IEEE 802.11 standard. This standard encompasses the lower layers of the OSI model for transport of data as ethernet frames using a spread spectrum based radio link.

This technology opens the possibility of building a network without having the problems associated with, and the cost of putting some sort of physical transmission medium in the ground. Instead, antennas can be used to send and receive the data using radio waves through free air. Because of the relative simplicity of the currently available commodity hardware that uses 802.11 technology it is relatively easy to build a local wireless community network in a town. Using this network people can share resources among each other. Furthermore, the network can connect to the Internet providing a low cost way of crossing the last mile to the user at high bandwidth and the possibilities of rolling out voice-over-ip (VOIP) technologies in connecting communitities together 


\section{Motivation}

The past decade has seen steady exponential growth of the Internet as well as cellular telephony. However, in terms of absolute numbers the growth is starkly behind that of the developed world. The difference is even more stark if we examine the density of access to communication technology (refer table1).

Parallel with the growth, the digital divide has grown too. The reasons for this are not hard to find. Communication evolution has been shaped by technical innovations and market forces in the western world. The business model thrives where the average percapita income is high (U.S. $\$ 20,000$ or more). For developing nations, the growth has been concentrated in metro pockets. This is especially unfortunate since the majority of the developing world population is in rural areas. Hence the low tele-density as given in Table1.

\section{Table 1}

\begin{tabular}{|r|r|r|r|}
\hline $\begin{array}{r}\text { Country/ } \\
\text { Region }\end{array}$ & $\begin{array}{r}\text { Telephone } \\
\text { (users/1000) }\end{array}$ & $\begin{array}{r}\text { Cell Phone } \\
\text { (users/1000) }\end{array}$ & $\begin{array}{r}\text { Internet } \\
\text { (users/1000) }\end{array}$ \\
\hline \hline U.S.A. & 664.5 & 450.8 & 501.5 \\
\hline Japan & 596.9 & 587.8 & 439.4 \\
\hline Taiwan & 573.4 & 968.8 & 349.0 \\
\hline Germany & 634.8 & 682.3 & 373.6 \\
\hline \hline Brazil & 217.8 & 167.3 & 46.6 \\
\hline China & 138.1 & 110.3 & 25.7 \\
\hline Africa & 26.2 & 32.2 & 8.5 \\
\hline India & 33.8 & 6.3 & 6.8 \\
\hline
\end{tabular}

Source : International Telecommunications Union Statistics. http://www.itu.int//TU-D/ict/statistics/

The cost of large-scale communication deployment is out of reach of developing countries. The cost per land-line telephone connection today is about U.S. $\$ 200$, and can be expected to drop to about $\$ 150$ in the next couple of years. This translates to a tremendous cost for a large-scale deployment, also $60-70 \%$ of the deployment cost is in the access network and not the core network. This acts as a further disincentive for rural deployment since rural population density is much lower than in cities. While cellular wireless technologies may help in quick deployment, like land-lines, their cost structure too is suited for the developed world. The service, and more importantly the equipment, are value-priced for markets where the users are willing to pay a high price.

In rural settings, the density of population as well as its paying capacity is small and hence a deployment cannot pay for itself. In this context, consider the 802.11 family of wireless technologies. Since its inception in 1994, 802.11 WiFi ha shown tremendous growth and acceptance (in US/Europe) as a lastmile wireless solution in corporate/enterprise settings as well as in home networks. With widespread acceptance of the technology, this has reached open/inter-operable standard, and become competitive.

The wireless community network built has a number of requirements to be sucessfull. First of all it has to be as "open" as possible to the users and to the developers. Being "open" enables authorized users within the community to actively use the network and participate in the building thereof. Another constraint is that the network should be reliable and low-cost at the same time. These constraints are met in a design using commercial off -the- shelf (COTS) and home built (low cost) hardware (network boards, 
antennas and PC hardware) components and open source software. (such as Linux or FreeBSD and other packages well known to the Free software community). However in the local context the wireless community network paradigm is yet to get off with a bang, partially due to the draconian rules of the incumbent telecom regulator and due the non availability of high degree of technical know-how within the communities in deploying and using new and emerging technologies.

Various wireless related technologies have been implemented in Sri Lanka over the past several years. However, during the last 10 years or so there has been an explosive growth in both the fixed and the mobile telecommunication market segments which has given rise to the availability of numerous value added data and ICT enabled wireless services. These services range from enabling technologies for peer-to-peer device access to broadband content delivery and internet access services more or less zeroing in and around the commercial capital Colombo and in metropolis. Service lines vary from voice enabled services to high speed DSL internet access. Though Metro Ethernet solutions are offered by the service providers concentrating mostly in the Colombo metropolitan areas, the service coverage is yet to reach the households and the rural areas. WiMAX pilot implementations has started with a bang but vendor equipment incompatibility, non availability of define standards for equipment interoperability, high cost of CPE (Customer premises equipment) would be a discouraging factor for rural area deployment. However as an alternate to fiber driven backbone links, service providers could use WiMAX within their backbone networks, which we anticipate will happen in the local context.

However the rural communities are largely neglected in the local context for provisioning of internet and other value added data services. Most of the incumbent service providers are catering only to voice enabled services while they cover majority of the areas in Sri Lanka, in some cases even the voice services are not available to some rural communities which effectively isolate them from the happenings of the rest of the world. The authors aim to provide a blueprint of a proven but not yet locally implemented delivery infrastructure, primarily targeting the internet access and for provisioning other value added internet protocol related services to the rural masses.

\section{Method}

While taking into consideration the prevailing set of procedures and rules set by the local telecom regulator, it is more or less impossible to setup a community based wireless networking infrastructure using the ISM 2.4G $\mathrm{Gz}$ and the $5.1 \mathrm{Ghz}$ band since these bands are yet to be made license free (for outdoor use) in the local context to be freely used $b$ the public. Hence most of the content of this paper maps out a strategy to be used the moment the ISM band is made freely available to the public use (for outdoor) in the Sri Lankan context, which the authors envisage would happen quite soon.

\subsection{Viable Technologies}

A number of different technologies are available to build wireless computer networks. Most commercial solutions are proprietary to certain vendors or do not use low cost hardware. The IEEE 802.11 standard (called WiFi in the commercial world) allows users to network their machines using radio technology. 
Different substandards have formed specifying the bandwidth or radio frequency the networks operate on. The standard defines a number of operation modes which allow for adhoc, point to point and point to multi point networking.

Though the standard calls for two transmission technology standards, Direct Spread Spectrum (DSS) and Frequency Hopping Spread Spectrum (FHSS), the market has by and large standardized on DDS for indoor and outdoor point to multipoint use. FHSS, which is more robust against certain types of interference and densely packed endpoints, is currently only seen on long point to point connections where interference and frequency allocations are at a premium, for example at an aggregation point. In this study we have chosen the $802.11 \mathrm{~g}$ as primary standard mostly because of the availability of equipment, open source drivers and the cost of the hardware. IEEE $802.11 \mathrm{~g}$ is a DSS radio technology supporting link speeds of $1,2,5.5$ and $11,54 \mathrm{Mbit} / \mathrm{s}$. The standard is defined for home and office use, but with special measures (antennas) it is also applicable for crossing longer distances outdoors. (maximum of approximately 15 $\mathrm{Km}$ in a line of sight). Appropriate off the shelf low cost but reliable wireless access points together with the technical creativity could be amply used in creating short and long distance wireless networks for information exchange without much of a capital expenditure involved.

\subsection{Topology}

\subsubsection{Pre-planning}

In this paper we explore the possibilities of using WiFi cost-effectively in sparsely populated and rural areas. We use a 'life cycle' approach, considering both capital and operations costs which should be done over a prolonged study period. Our model takes into account market demand and segmentation, customer adoption and retention rates, service pricing, as well as technology-based factors such as range, bandwidth, antenna gain, receiver sensitivities and signal strengths. To make the assessment realistic, we model a real rural area: Mahavilachchiya in the district of Anuradhapura in Sri Lanka, which has a population of nearly 20,00 of whom around 8,000 live near the Horizon Lanka Foundation (www.horizonlanka.org ). The land area is virtually flat and a fitting environment for deploying a wifi enabled wireless network in offering NEAR broadband access to all.

\subsubsection{Deployment}

We consider first a baseline case where the network consists of APs serving end users in a point-to-multipoint type configuration which is interconnected to switches or routers using point-to-point wireless back haul links. The Network we anticipate in deploying and operating would be situated closer to the Horizon Lanka Project Office and we believe establishing an effective coverage area of 25 square $\mathrm{Km}$ would be possible.

The thrust of the system is to provide secure outdoor wireless access with a reasonable quality of service factor for accessing internet resources and peer communications. From a household a stationary antenna should preferably have a line of sight to the nearest network access point. As the maximum distance between the client and the network access point (a network node) is limited due to the RF power output limits imposed by the regulator, the Network we are envisaging is to operate within a coverage area of 25 square $\mathrm{Km}$, which covers a substantial area of our intended terrain. Combining this knowledge with the anticipated traffic and bandwidth needs, it shows that we need multiple nodes distributed over the coverage area. The nodes themselves have to be interconnected.

The interconnection of the nodes is also using wireless links, making the network completely 
independent of the local (wired) infrastructure and thus very cost-efective and without significant monthly or other regularly repeating costs. A mesh between the nodes will be formed, as each node is connected to other nodes by at least 2 different connections. With this approach, adding extra nodes to the network will add redundant paths and therefore will increase the total bandwidth. The topology is comparable to cellular telephony. Cells for users are created. In these cells users share the total available bandwidth. The cells are interconnected by point-to-point wireless connections

\subsubsection{Radio Planning}

There is an availability of 13 channels allowed in the $2.4 \mathrm{GHz}$ frequency band to run radio networks, however in the local context $2.4 \mathrm{Ghz}$ band is yet to be made free for public use . In Figure 1 it is shown that there are 3 completely separate channels available. Combining this knowledge with the topology and the goal of providing coverage in a fairly big area poses a challenging problem The problem is solved by careful selection of the channels, antennas, and the location and polarisation thereof. Also interferrence of the available channels on the radio level is a problem. The $2.4 \mathrm{GHz}$ band is in use. The use of WiFi in this so-called ISM (Industrial, Scientific, Medical) band is unlicensed, provided the above mentioned channel restrictions are obvserved and the efective output power of the antenna does not exceed $20 \mathrm{dBm}(100 \mathrm{~mW})$.

Using radio-planning software we can simulate the propagation of the signal and optimize the location of the different sites. Nevertheless, a site survey is always needed to measure the noise generated by other radio sources and check the signal strength of the already running nodes. Due to the high absorbance of the radio signals by for example trees in the line of sight, local measurements are essential in planning a node.

Figure1

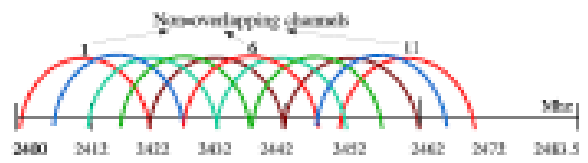

Overlapping 802.11 channels: schematic

Figure 2

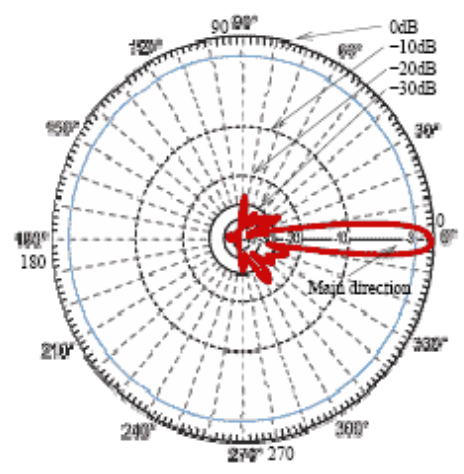

Spatial radn. pattern of parabolic grid antennae

Bridging the Digital Divide by Building... 


\subsubsection{IP Addressing and Address space allocation}

The network uses TCP/IP as transport layer, therefore every active element in the network should have an address. Using a private IP version four range enough addresses will be available. Using IP version six will be a future enhancenment and is not yet implemented. So as the IP network grows there is a need for not only planning the radio frequency space, but also planning the IP space. The network is using a private IP range. Network address transaltion schemes may be needed to be used if services such as hosting of web sites, virtual mail server hosting is required. Though this requirement is not anticipated in the near future, with the growth of the network and the increasing presence on the internet, we believe the requiremnet would occur on a on demand basis.

\subsubsection{Site Allocation}

The topology we would be outlining would have one central distribution facility with stratergically placed bakchaul nodes scattered around the perimeter.Once a site is designated by using the planning procedure, it might be a tedious task to get permission of the different building-owners to have the antennas and equipment installed. Since locations with high degree of ground clearance and visibility is of paramount importance and due to self sustaining nature of the network, the relevant stakeholders should be educated on the delieverables of the project and the impact on the community at large. It is asumed that a place of worship (temple, kovil etc) or a school should be chosen to host the main distribution facility (equipped with the necessary backhual links and other relevant communication gear). Availability of electricity is of prime importance and care should be given for hosting the equipment securely. Also co-operating commercial enterprises that want to provide services on the network, or want to make use of the network for private communications too should be encouraged to participate in selecting a site providing the initial ground work for the sustanability of the network infrastrcuture.

\subsubsection{Setting up the Infrastructure}

The typical network node setup consists of a number of directional antennas and a computer sub system, which could be used as proxy server(s) for content caching, mail servers and domain name servers (DNS), web servers, etc. It is highly recommeneded to use open source components for all the server modules.

The number of directional antennas would depend on the size of the area to be covered and also the number of repeater sites that would be in and around the perimeter. As for the central distribution wireless access point which is the central nervous system of the entire topology, we encourage to look at low cost wireless solutions which are available from an array of vendors, and to never lock horns with a proprietary vendor solution. The selected main distribution access point(s) should exhibit the following key characteristics :

- Powerful QoS control mechanisms

- $\quad$ Peer-to-Peer (P2P )traffic filtering

- High availability with VRRP or other

- Support for bonding of Interfaces

- Stateful firewall, tunnels and support for IPsec

- $\quad$ STP bridging with filtering capabilities

- $\quad$ Super high speed $802.11 \mathrm{a} / \mathrm{b} / \mathrm{g}$ wireless with WEPMPA 
- WDS and Virtual access point features

- $\quad$ Support for RIP, OSPF, BGP routing protocols

- $\quad$ Remote GUI and Web administration capabilities

- Real-time configuration and monitoring capabilities

- Supporting mesh network infrastructure to seamless handover of clients from one AP to another in an event of a failure.

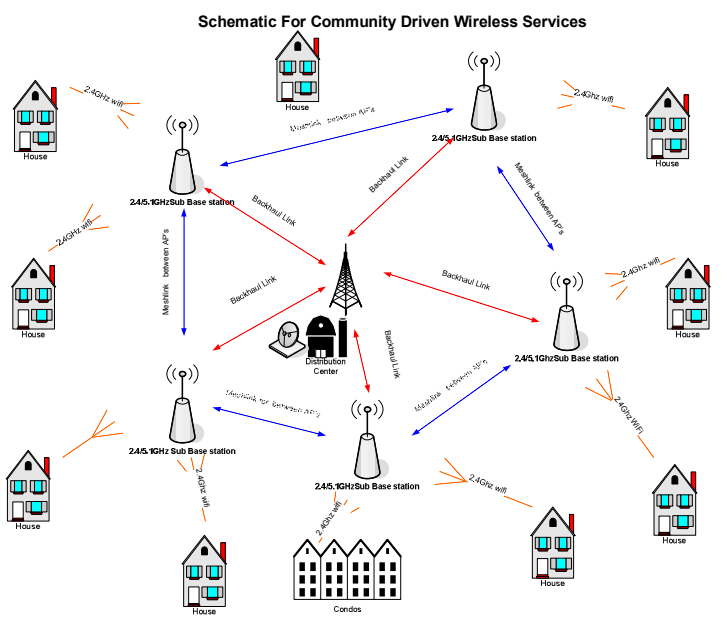

Figure 3

Figure 3 illustrates a typical layout of the distribution architecture. At the client site (i.e typical household) a pc equipped with an off the shelf $802.11 \mathrm{~b} / \mathrm{g}$ wireless card with relevant software drivers loaded together with a yaggi type directional outdoor antenna (a gain of at least 9-10dbi) would be sufficient to attract the signals and connect to the network. Care should be given to induce some sort of a lightning protection mechanism to the outdoor antenna, which could be susceptible to lightning strikes. The overall cost of this setup would be less than 100USD and we believe the domestic outdoor antenna should be manufactured with the local expertise.

\subsubsection{Securing the Network}

Apart from the physical security at the main facility, we propose to use IEEE $802.1 \mathrm{x}$ WEP based security with the integration of a central RADIUS (Terminal Access Controller Access-Control System) or a TACACS (Terminal Access Controller AccessControl System) server to authenticate the valid client connections. This setup can be used to secure the network against unauthorized roaming users. Also users would be educated on the security aspects where ever possible and elementary measures they should consider in order to prevent any data security related issue.

The nature of the OSI transport layer adds an extra possibility of Denial of Service (DoS) attacks by jamming a connection on the radio level. Because we are operating concurrently with other users in the same frequency space this can be a problem. Adding redundant paths together with the appropriate routing protocols is the way to overcome the issue. 


\section{Results}

\section{Applications}

Apart from provisioning internet access from the central site infrastructure, pee to peer connectivity is strongly encouraged and made possible with internet like technologies. Schools could be linked with each other and curriculum and subject matters could be discussed in details while students could collaborate with each other remotely, via email and other real time voice and video enabled applications. Where ever possible client sites are guaranteed bandwidths of greater than $512 \mathrm{Kbps}$ and we believe throughputs of at least $6 \mathrm{Mbps}$ are achievable in most of the locations to the backbone which would provide a satisfactory level of operations to carry out most of the bandwidth intensive tasks.

We believe VOIP enabled application would play a major role in this infrastructure, to start up VOIP services enabled as an alternative to the common wired and WLL based telephony connections, using either soft phones or low grade IP handsets. Subscribers could make calls to each other without any cost involved at all. By inducing the OpenSource PBX solution by Asterisx (www.asterisk.org) or by OpenPBX (www.openpbx.org) a robust VOIP infrastructure could be built on the existing wireless network foundation. Off course the modalities of the operation should be agreed upon but the important factor is that it is achievable at a fraction of a cost. As shown in Figure 4, a centrally hosted PBX solution which is integrated with a telecom link (a channalized voice E1 connection) from a telecom provider could be used for distributing the VOIP based telephony connections to the subscribers. While number planning and other related call forwarding issues have to be worked on, the authors believe that this process is achievable and would deliver much awaited telephony solution to the rural areas.

Figure 4

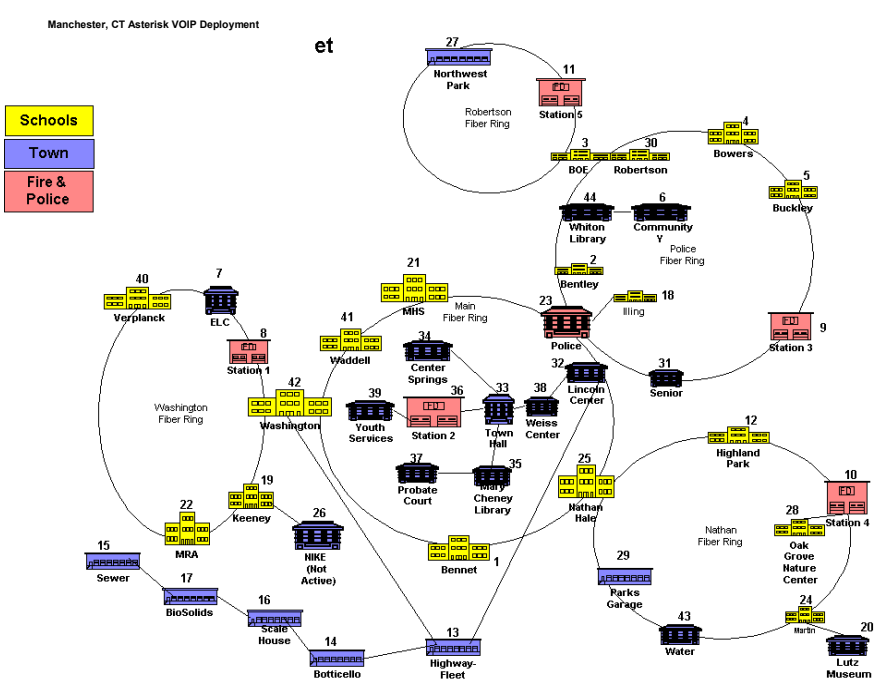




\section{Issues}

Problems that were encountered during the startup phase of the network can be divided into two groups. These were technical and non-technical problems. Technical problems are seen on every level of the network stack. Starting with the physical level, it is difficult to plan the network using the different constraints like noise, limited channels available and natural obstacles. Due to this problem the configuration of the network is constantly evolving. Changing of antenna directions can be difficult once a node is set up. On the higher levels we have encountered problems with the network drivers for the wireless boards we are using. These problems can seriously affect the throughput and reliability of the network. Here the use of open source software and the open source development model pays off. Problems can be solved within the group, or with help and information gained from the Internet.

Also having a choice between different solutions to problems helps. On the IP level having many point-to-point links and a number of applications results in quite a big puzzle to get everything configured correctly. Using a central configuration repository and the use of routing protocols helps, but some problems still remain to be solved. For example when connecting to the Internet (with a number of providers) a routing and numbering issue needs to be solved. Also the latency might pose a problem in some applications. NAT (Network Address Translation) and DHCP services could be used which may lead to incurring operational issues in deployment of voice over IP solutions. Furthermore, sometimes the reliability of the hardware can be a problem. Because of cost issues complete (used) PC machines are in use as network nodes. These machines are not as reliable as dedicated routers which are built as dedicated embedded systems without moving parts (like fans or hard disks).

Minimizing the points of failure, testing, validating and proper maintenance helps a lot here. Currently such a small embedded system is in development to assure better reliability and enhance the mean time between failure of a network node. The main issue is that in the local context such systems are yet to gain familiarity within in the local ICT environment. Non-technical problems are gaining acceptance within the town and the community. Without a broad acceptance and support of various organizations it is not possible to build this kind of a network in a not-for-profit fashion. Access to rooftops of high buildings etc is essential. However, we do not believe that a single entity has any chance of succeeding at all. The upfront costs of building a network without volunteers are very large. Possible revenue streams will be insufficient to recoup some degree of expenditure and maintenance cost.

\subsection{Related work}

In many parts of the world wireless communities are developing. In the USA there are a number of leading initiatives [Bawug, Seattlewireless, FreeNetworks]; also in Europe and Australia communities have been formed. The main difference between the Wireless network initiatives taking place in the local context and what is happening in the other parts of the world is that while the world is moving ahead on the deregularization of the ISM band and giving communities free access to wireless technologies, draconian rules prevail in Sri Lanka which up to now prohibit the use of ISM band for outdoor wireless access, thereby depriving the communities to use innovative technologies for the betterment of life. Another major plus point is that the infrastructure will be based on open standards and open source software, that shall free us from vendor lock-ins and is achieving the broadest possible range of applications while providing sustainability of the complete system. 


\subsection{Outlook}

We did test the network for its full operation, and the corresponding applications were successfully deployed, innovative applications such as Skype (www.skype.com) too were integrated to the environment to place international calls at low cost rates. Due to the rather small group of technically skilled people the main focus of Wireless setup has been to test run the system, since the needed legal infrastructure is yet to prevail in the local context form deploying outdoor wifi connectivity. In addition we have concentrated on knowledgeable individuals and professional organizations as primary test users, because they require less IT-assistance from the volunteers. As this is accomplished, the next step is extending the network to the suburbs and connecting the actual private users to the network. We believe to replicate this model with the help of the necessary ICT governing bodies in Sri Lanka.

While new technologies are being tested to cope with the expected growth of the network, also in evaluation is the use of more complex and advanced technologies using mesh or ad-hoc networking. The use of applications that require Quality Of Service facilities in the network such as IP telephony and wideband video streaming is being studied. Engaging in research in this environment is very attractive.

\section{Conclusion}

With the use of relatively low cost technologies and open source software it is possible to build a wireless network which is used by the community. Technical problems do exist, but with the use of open source software and low cost proven hardware solutions these can be solved. The process of building a network using a loosely-coupled group of volunteers is not easy but bringing organization to the group when the network becomes more complex helps a lot. Having the back-up of the community and local enterprises also helps to gain momentum and visibility of the project, which in return speeds up the implementation. We believe wireless networking and Wifi in general has a lot of potential in the Sri Lankan context in bridging the digital divide effectively.

\section{References}

BaWUG, The Bay Area Wireless Users group, http://www.bawug.com

Int. Journal of Electronics and Communications,54-4 2000 pg. 211-217.

Free Networks, information on different community networks, http://www.freenetworks.org/

Hu, Y., A. Perrig, D.B. Johnson, Ariadne, A. 2002. Secure On-Demand Routing Protocol for Ad-Hoc Networks., MobiCom 2002.

Malhotra, R. 2002. IP Routing, O'Reilly \& Associates Inc.

Flickenger, Rob. 2001. Building Wireless Community Networks, O'Reilly \& Associates Inc.

Gast, M. 2002. 802.11 WirelessNetworks, O'Reilly \& Associates Inc.

Horizon Sri Lanka, www.horizonlanka.org

The Economist Technology and Development -The real digital divide, http://www.economist.com/opinion/displayStory.cfm?story id=3742817 